Research Paper

\title{
Prognostic Value of Lymphocyte Activation Gene-3 (LAG-3) Expression in Esophageal Squamous Cell Carcinoma
}

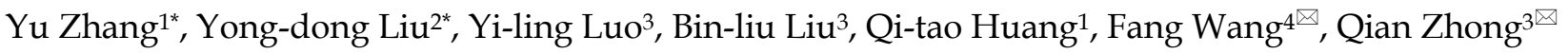 \\ 1. Department of Pathology, Sun Yat-sen University Cancer Center, No. 651 Dongfeng East Road, Guangzhou 510060, China \\ 2. Department of Pathology, The First Affiliated Hospital of Sun Yat-sen University, No. 58th Zhongshan 2nd Road, Guangzhou 510080, China \\ 3. State Key Laboratory of Oncology in South China, Department of Experimental Research, Sun Yat-sen University Cancer Center, No. 651 Dongfeng East \\ Road, Guangzhou 510060, China \\ 4. Department of Molecular Diagnosis, Sun Yat-sen University Cancer Center, No. 651 Dongfeng East Road, Guangzhou 510060, China \\ *Equal contributors \\ $\square$ Corresponding authors \\ (c) Ivyspring International Publisher. This is an open access article distributed under the terms of the Creative Commons Attribution (CC BY-NC) license \\ (https://creativecommons.org/licenses/by-nc/4.0/). See http://ivyspring.com/terms for full terms and conditions.
}

Received: 2018.04.28; Accepted: 2018.08.26; Published: 2018.10.20

\begin{abstract}
Esophageal squamous cell carcinoma (ESCC) is a malignant epithelial tumor with a high incidence in East Asia and the Middle East. The outcomes for ESCC patients are usually not optimal due to the recurrence and metastasis. This study is aim to examine the expression and the prognostic value of LAG-3 in ESCC. We applied immunohistochemistry analysis to examine the expression of LAG-3, CD4 and CD8 in 287 ESCC cohorts. Our study demonstrated that the decreased LAG-3 expression was significantly associated with CD4 tumor-infiltrated lymphocytes (TILs) $(p=0.000)$, CD8 TILs $(p=0.000)$, and the advanced clinical stages $(p=0.041)$ by Chi-square analysis. Kaplan-Meier survival analysis revealed that higher LAG-3 expression were positively correlated with a better overall survival (OS) $(p=0.010)$ and better progression free survival (PFS) $(p=0.006)$, especially in the patients at stages T1-2 status ( $p=0.001, \mathrm{OS} ; p=0.001, \mathrm{PFS}), \mathrm{N} 0$ status $(p=0.036, \mathrm{OS} ; p=0.050$, PFS), and early stages (I-II) $(p=0.006$, OS; $p=0.008$, PFS). Both high of CD4 TIL /CD8 TIL ratio and LAG-3 expression were correlated with longer OS and PFS. Cox proportional hazards regression analysis showed that LAG-3 is an independent biomarker of survival (HR, 0.724; 95\% Cl 0.526-0.995; $p=$ $0.047)(p=0.036)$. Taken together, we found that high expression of LAG-3 was correlated with an improved survival and LAG-3 is an independent predictor of survival, suggesting that LAG-3 may serve as a useful immune marker for the prognosis of ESCC.
\end{abstract}

Key words: LAG-3, ESCC, TIL, prognosis

\section{Introduction}

Esophageal carcinoma (EC) is one of the common causes of cancer-related death worldwide. There are two major subtypes of $\mathrm{EC}$, which are esophageal squamous cell carcinoma (ESCC) and esophageal adenocarcinoma (EAC) [1]. ESCC is the main histological subtype of EC in East Asia and the Middle East [2]. The current treatments for ESCC are still surgery, chemotherapy and radiotherapy. Although these conventional therapies achieved certain effects, the outcomes of ESCC patients are not significantly improved. Therefore, it is urgent to look for efficient molecular predictors and alternative therapeutic ways for ESCC.

Recently, the tumor microenvironment gains the increasing interests for developing potential antitumor strategies in cancer field. There were many evidences showing that the increasing number of tumor-infiltrating lymphocytes (TILs) in $\operatorname{ESCC}[3,4]$. Co-inhibitory or immune checkpoint receptors play essential roles in tumor immunity escape. Therefore, 
blocking of co-inhibitory or immune checkpoint receptors might achieve potential anti-tumor immunity. For example, blockade of programmed death 1 (PD-1) expressed in exhausted T-cell has been shown a good clinical efficacy in the patients of melanoma, non-small cell lung cancer, and ovarian cancer et al [5-8].

Lymphocyte activation gene-3 (LAG-3) has been demonstrated as an important immune checkpoint in tumor immunity. LAG-3 is expressed on TILs [9], natural killer (NK) cells [9] and B cells [10]. LAG-3 have been shown to bind three ligands, major histocompatibility complex (MHC) II [11], LESTin [12] and Galectin-3 [13]. It has been reported that the expression of LAG-3 was correlated with an improved survival in both non-small-cell lung cancer (NSCLC) patients [14] and microsatellite instability-high (MSI$\mathrm{H})$ colon cancer patients [15]. Additionally, blocking LAG-3 and PD-1 induced CD8 $T$ cells killing activation in tumors [16]. PD-1, $\mathrm{T}$ cell immunoglobulin-3 (TIM3), and TIGIT (T cell immunoglobulin and ITIM domain) were shown to be upregulated on TILs in ESCC patients, and PD-1 expression on TILs was correlated with late T status in this ESCC cohort [17].However, the role of LAG-3 in ESCC still remains elusive. In this current study, we investigated the expression of LAG-3 and analyzed its prognostic values in ESCC patients.

\section{Material and Methods}

\section{Patients}

Two hundred and eighty-seven paraffinembedded ESCC specimens were collected at Sun Yat-sen University Cancer Center. All the ESCC patients were clinically diagnosed and obtained during radical surgery prior to radiotherapy or chemotherapy from 2000 to 2006. Tissues microarrays were constructed from each specimen. The follow-up time for survivors was from 26 to 5137 days (median 1225 days). Clinical stages were defined based on the 7th edition of the Union for International Cancer Control-TNM (UICC-TNM) Classification. This study was approved by the Institutional Research Medical Ethics Committee at SYSUCC. The clinical characteristics of the ESCC patients were presented in Table 1.

\section{Immunohistochemistry staining}

The 4- $\mu \mathrm{m}$ Formalin-fixed, paraffin-embedded ESCC tissue microarray sections were deparaffinized in xylene and with an alcohol gradient to rehydrate. The sections were immersed with three percent hydrogen peroxide to block endogenous peroxidase activity at room temperature for 10 mins, then boiled in Citrate Antigen Retrieval Solution $(\mathrm{PH}=6.0)$ for 5 mins in a microwaver for antigen retrieval. After that, the sections were incubated with the LAG-3 antibody (diluted at 1:150, LifeSpan BioSciences, Inc. Seattle, WA, U.S.), CD4 antibody (diluted at 1:100, ZSGB-BIO, Beijing, China), CD8 antibody (diluted at 1:100, ZSGB-BIO, Beijing, China) in a wet chamber for one and half hrs at $37^{\circ} \mathrm{C}$. During the next day, the sections were washed with PBS three times and then incubated with a secondary antibody for 30 mins at $37^{\circ} \mathrm{C}$. After that, the sections were stained with 3,3diaminobenzidine for 2 mins and then counterstained with Mayer's hematoxylin to stain nucleus. At the end, the sections were dehydrated and mounted.

Table 1. Clinicopathologic characteristics of 287 patients

\begin{tabular}{ll}
\hline Clinicopathologic parameters & Cases (\%) \\
\hline Age & \\
Median (57) & \\
Range (32-81) & \\
$\leq 57$ & $150(52.3)$ \\
$>57$ & $137(47.7)$ \\
Gender & \\
Male & $212(73.9)$ \\
Female & $75(26.1)$ \\
T status & \\
T1-2 & $83(28.9)$ \\
T3-4 & $204(71.1)$ \\
N status & \\
N0 & $152(53.0)$ \\
N1/2/3 & $135(47.0)$ \\
M & \\
No & $281(97.9)$ \\
Yes & $6(2.1)$ \\
Stage & \\
I-II & \\
III-IV & $174(60.6)$ \\
Grading & $113(39.4)$ \\
G1 & \\
G2 & $51(17.8)$ \\
G3 & $166(57.8)$ \\
Recurrence & $70(24.4)$ \\
No & \\
Yes & $274(95.5)$ \\
Metastasis & $13(4.5)$ \\
No & \\
Yes & $207(72.1)$ \\
\hline
\end{tabular}

\section{Evaluation of Immunohistochemistry}

The immunoreactivities were scored by two independent pathologies blinded to the clinical characteristics of these ESCC patients. In the tumor region, LAG-3, CD4, and CD8 were scored as the percentage of TILs. An optional cut-off value for high and low expression was determined by the median of the score of LAG-3, CD4, and CD8, respectively. All the ESCC were divided into two subgroups according to the median score of LAG-3 immunostaining. 


\section{Statistical analysis}

Statistical analysis was performed using SPSS software version 19.0. The correlation between LAG-3 and clinicopathological status of ESCC patients was examined by Spearman's rank correlation analysis and Chi-square test. Both progression-free survival (PFS) and overall survival (OS) curves for both high and low expression of LAG-3 were plotted by KaplanMeier analysis and log-rank test. Univariate and multivariate regression analysis were conducted using Cox proportional hazards regression model. A $p$-value $<0.05$ was considered as statistically significant.

\section{Results}

\section{Patient characteristics}

The clinical and histopathologic data for all 287 ESCC patients are descried in Table 1 . The median age was 57 (range from 32 to 81 ). Around $71.1 \%$ of ESCC patients are at late $\mathrm{T}$ status (T3+T4), and $47 \%$ patients have lymph node metastasis. According to the UICC guideline, there were $39.4 \%$ patients are at late clinical stages (III+IV). About $17.8 \%, 57.8 \%$, and $24.4 \%$ patients were diagnosed with well differentiated (G1), intermediately differentiated (G2), poorly differentiated ESCC (G3) based on their histopathological grades. There were 85 patients were found with either local recurrence $(n=13)$ or distant metastasis $(n=80)$ during the follow-up time.

\section{Association of LAG-3 expression with clinicopathological features}

To determine whether LAG-3 expression was correlated with clinicopathological features, all the ESCC patients are divided into high and low LAG-3 expression groups. In our study, the cut-off score for the expression of LAG-3 was set at 0.001. If ESCC patients with negative staining intensity for LAG-3 were designated as low expression of LAG-3; if ESCC patients with visual staining intensity for LAG-3 were defined as high expression of LAG-3 (supplemental figure 1). High expression of LAG-3 was observed in $172 / 287(59.9 \%)$ of ESCC patients. LAG-3 expression was significantly associated with clinical stages $(p=$ 0.041). Lag-3 expression was correlated with CD4 TIL $(p=0.000)$ and CD8 TIL $(p=0.000)$, similarly to the other reports $[18,19]$.

High numbers of CD4 TIL and CD8 TIL have been reported in ESCC $[3,4]$. In our study, the number of CD4 TIL was negatively correlated to $\mathrm{N}$ status in ESCC patients (supplemental Table 1) $(p=0.044)$. The number of CD8 TIL (supplemental Table 2) and the ratio of CD4 TIL/CD8 TIL (supplemental Table 3) were not associated with any clinicalpathological parameters of these ESCC patients. Furthermore, CD4 TIL/CD8 TIL is also positively correlated with LAG-3 expression $(\mathrm{p}=0.019$, Table 2$)$.

Table 2. Relationship between LAG-3 expression and various patient characteristics

\begin{tabular}{|c|c|c|c|c|}
\hline \multirow[t]{2}{*}{ Characteristics } & \multirow[t]{2}{*}{ No of patients } & \multicolumn{3}{|c|}{ Expression of Lag3 } \\
\hline & & Low & High & $P$ \\
\hline \multicolumn{5}{|l|}{ Patients } \\
\hline \multicolumn{4}{|l|}{ Age } & 0.980 \\
\hline$\leq 57$ & 150 & 90 & 60 & \\
\hline$>57$ & 137 & 82 & 55 & \\
\hline \multicolumn{4}{|l|}{ Gender } & 0.891 \\
\hline Male & 212 & 128 & 84 & \\
\hline Female & 75 & 44 & 31 & \\
\hline \multicolumn{4}{|l|}{ T status } & 0.468 \\
\hline $\mathrm{T} 1-2$ & 83 & 47 & 36 & \\
\hline T3-4 & 204 & 125 & 79 & \\
\hline \multicolumn{4}{|l|}{$\mathrm{N}$ status } & 0.142 \\
\hline No & 152 & 85 & 67 & \\
\hline $\mathrm{N} 1 / 2 / 3$ & 135 & 87 & 48 & \\
\hline \multicolumn{4}{|l|}{ M } & 0.617 \\
\hline No & 281 & 169 & 112 & \\
\hline Yes & 6 & 3 & 3 & \\
\hline \multicolumn{4}{|l|}{ Stage } & 0.041 \\
\hline I-II & 174 & 96 & 78 & \\
\hline III-IV & 113 & 76 & 37 & \\
\hline \multicolumn{4}{|l|}{ Recurrence } & 0.904 \\
\hline Yes & 274 & 164 & 110 & \\
\hline No & 13 & 8 & 5 & \\
\hline \multicolumn{4}{|l|}{ Metastasis } & 0.176 \\
\hline Yes & 207 & 119 & 88 & \\
\hline No & 80 & 53 & 27 & \\
\hline \multicolumn{4}{|l|}{ CD8 TIL } & 0.000 \\
\hline$\leq 0.035$ & 146 & 105 & 41 & \\
\hline$>0.035$ & 141 & 67 & 74 & \\
\hline \multicolumn{4}{|l|}{ CD4 TIL } & 0.000 \\
\hline$\leq 0.01$ & 152 & 109 & 43 & \\
\hline$>0.01$ & 135 & 63 & 72 & \\
\hline \multicolumn{4}{|c|}{ CD4 TIL/CD8 TIL } & 0.019 \\
\hline$\leq 0.3342$ & 173 & 96 & 76 & \\
\hline$>0.3342$ & 114 & 48 & 66 & \\
\hline
\end{tabular}

\section{Correlation of LAG-3 expression with overall survival}

Among 287 ESCC patients, there were a total 172 (59.9\%) cancer-related deaths at the final follow up. The five-year overall survival (OS) rate was $52.4 \%$ for this whole ESCC cohort (Figure 1A). The patients exhibiting a high LAG-3 expression presented a significantly longer OS (median, high vs low: 2202 vs 2664 days, log-rank test $p=0.010$ ) (Figure 1B). Further stratification of patient groups based on $\mathrm{T}, \mathrm{N}$, and clinical stages displayed that the correlation of high LAG-3 expression and higher OS was statistically significant in T1-2 status patients (median, high vs low: 2961 vs 1011 days, log-rank test $p=0.010$ ) (Figure $1 C$ ), N0 status patients (median, high vs low: 2703 vs 2361 days, log-rank test $p=0.038$ ) (Figure 1E), and clinical stages I-II patients (median, high vs low: 2244 vs 1691 days, log-rank test $p=0.006$ ) (Figure 1G) with ESCC. 

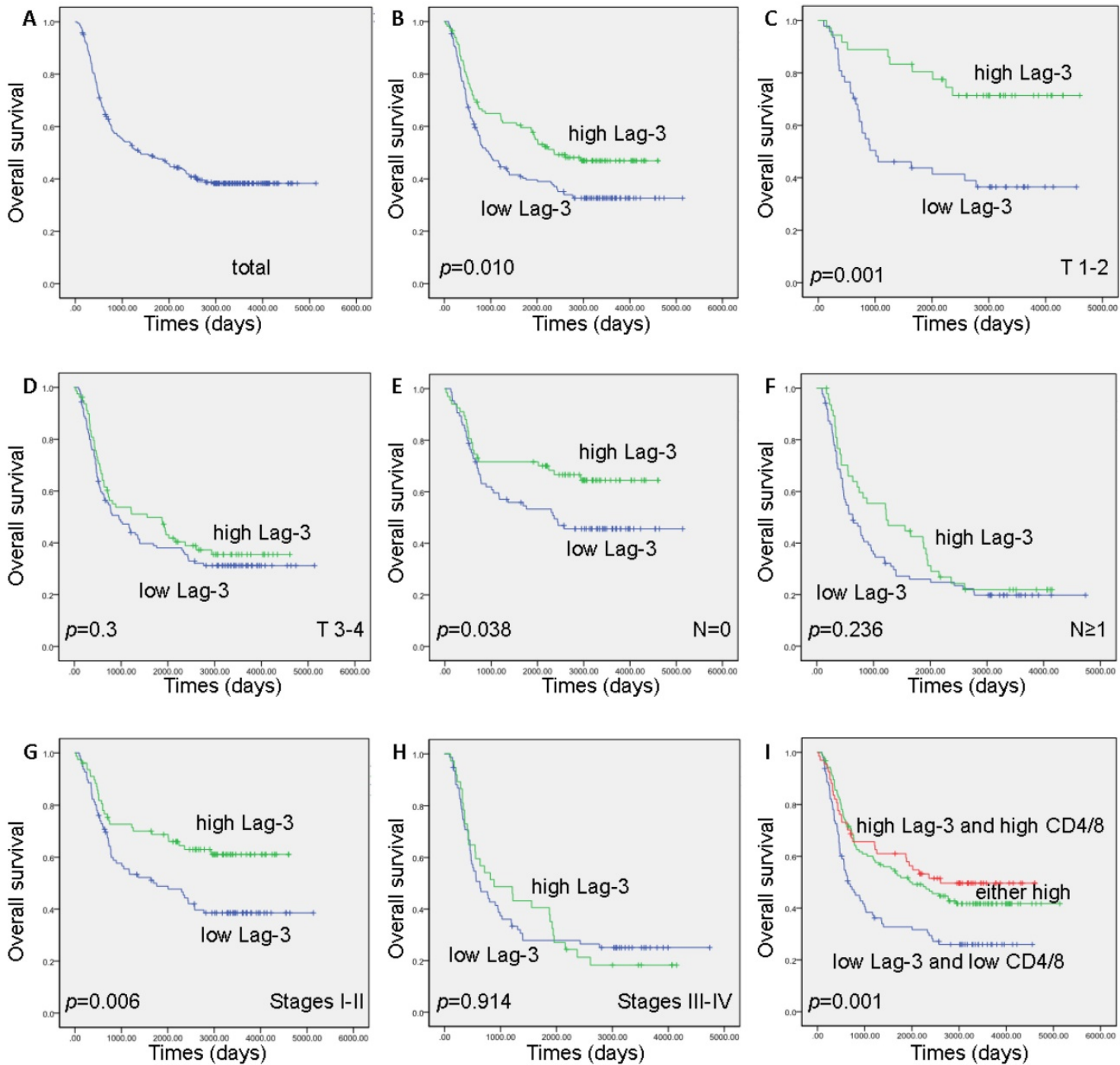

Figure 1. Kaplan-Meier survival curve and log-rank test analysis showing the association between LAG-3 expression and overall survival of ESCC patients. A. The five-year overall survival (OS) rate was $52.4 \%$ of 297 ESCC patient; B. High LAG-3 expression level was significantly correlated to OS $(p=0.010)$ in all ESCC patients; C. High LAG-3 expression was significantly associated with OS $(p=0.001)$ in ESCC patients at T 1-2 status; D. No significant difference in OS rate was found between LAG-3 high-expression and low-expression in ESCC patients at T 3-4 status; E. High LAG-3 expression was significantly associated with OS $(p=0.038)$ in ESCC patients at N0 status; F. No significant difference in OS rate was found between LAG-3 high-expression and low-expression in ESCC patients at $\mathrm{N} \geq 1$ status; $\mathbf{G}$. High LAG-3 expression was significantly associated with OS $(p=0.006)$ in ESCC patients at Stages I-Il; $\mathbf{H}$. No significant difference in OS rate was found between LAG-3 high-expression and low-expression in ESCC patients at Stages III-IV; I. Both high of CD4 TIL /CD8 TIL ratio and LAG-3 expression were correlated with longer survival $(p=0.001)$.

The patients presenting high CD4 TILs seemed to have a longer OS but this correlation did not have a significant statistic difference (median, high vs low: 1942 vs 994 days, log-rank test $p=0.184$ ) (Supplemental Figure 2A). However, high CD8 TIL was not associated with OS (median, high vs low: 1885 vs 1225 days, log-rank test $p=0.696$ ) (Supplemental Figure 2B). The patients having a high CD4 TIL/CD8 TIL were correlated with a significantly improved OS (median, high vs low: 2299 vs 906 days, log-rank test $p=0.015$ ) (Supplemental Figure 2C). Combining of CD4 TIL /CD8 TIL ratio with LAG-3, both high of CD4 TIL /CD8 TIL ratio and LAG-3 expression were correlated with longer survival, whereas low of CD4 TIL /CD8 TIL ratio and
LAG-3 expression were correlated with shorter survival (median, high, moderate vs low: 2607, 1956 vs 649 days, log-rank test $p=0.001$ ) (Figure 1I).

In addition, we stratified the patients into two subgroups according to $\mathrm{T}$ status and stages. Interestedly, high CD8 TIL seems to have a correlation with good prognosis at T 1-2 status (supplemental figure $3 \mathrm{~A}$ ) and at early stages (supplemental figure 3C) but with a worse survival at late stages (supplemental figure 3D), suggesting that CD8 TIL exhaustion might play an important role in tumor progress at late stages. A large scale of ESCC samples or flow cytometry analysis of exhausted CD8 TIL will be further investigated. 

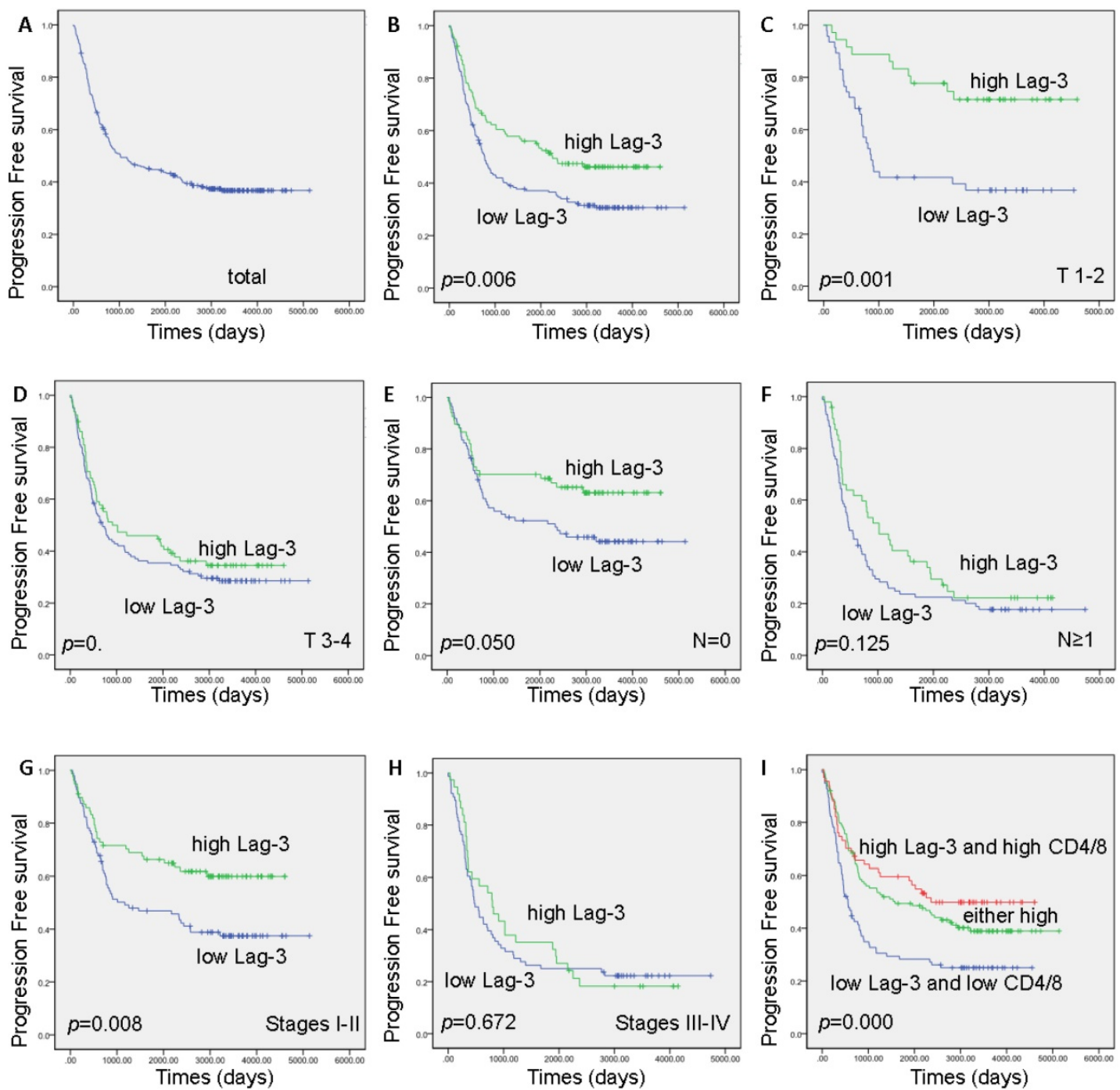

Figure 2. Kaplan-Meier survival curve and log-rank test analysis showing the association between LAG-3 expression and progression free survival of ESCC patients. A. The five-year progression free survival (PFS) rate was $55.3 \%$ of 297 ESCC patient; B. High LAG-3 expression level was significantly correlated to PFS $(p=0.006)$ in all ESCC patients; C. High LAG-3 expression was significantly associated with PFS $(p=0.000)$ in ESCC patients at T $1-2$ status; $\mathbf{D}$. No significant difference in PFS rate was found between LAG-3 high-expression and low-expression in ESCC patients at T 3-4 status; E. High LAG-3 expression was significantly associated with PFS $(p=0.050)$ in ESCC patients at N0 status; F. No significant difference in PFS rate was found between LAG-3 high-expression and low-expression in ESCC patients at N $\geq 1$ status; G. High LAG-3 expression was significantly associated with PFS ( $p=0.008$ ) in ESCC patients at Stages I-II; $\mathbf{H}$. No significant difference in PFS rate was found between LAG-3 high-expression and low-expression in ESCC patients at Stages III-IV; I. Both high of CD4 TIL /CD8 TIL ratio and LAG-3 expression were correlated with longer survival $(p=0.000)$.

\section{Correlation of LAG-3 expression with progression-free survival}

A total $177(61.7 \%)$ cancer-related progression were found at the final follow up. The five-year progression-free survival (PFS) rate was $55.3 \%$ (Figure 2A). A high LAG-3 expression exhibited a significantly longer PFS in this ESCC cohort (2050 vs 2571 days, log-rank test $p=0.006$ ) (Figure 2B). Furthermore, the correlation of high LAG-3 expression and improved PFS was statistically significant in T1-2 status patients (median, high vs low: 2903 vs 859 days, log-rank test $p=0.001$ ) (Figure 2C), N0 status patients (median high vs low: 2787 vs 2331 days, log-rank test $p=0.050$ ) (Figure 2E), and clinical stages I-II patients (median, high vs low: 2592 vs 1168 days, log-rank test $p=0.000$ ) (Figure 2G) with ESCC.

Although the patients with higher CD4 TILs seemed to have a longer PFS, this correlation did not have a significant statistic difference (median, high vs low: 1885 vs 816 days, log-rank test $p=0.164$ ) (Supplemental Figure 2D). High CD8 TILs was not associated with PFS (median, high vs low: 911 vs 1027 days, log-rank test $p=0.727$ ) (Supplemental Figure 2E). Interestingly, a high CD4 TIL/CD8 TIL ratio was correlated with a significantly longer PFS in these ESCC patients (median, high vs low: 2171 vs 772 days, log-rank test $p=0.009$ ) (Supplemental Figure 2F). Moreover, both high of CD4 TIL /CD8 TIL ratio and LAG-3 expression were associated with longer PFS, 
whereas low of CD4 TIL /CD8 TIL ratio and LAG-3 expression were associated with shorter PFS (median, high, moderate vs low: 2361, 1578 vs 557 days, log-rank test $p=0.000$ ) (Figure 2I).

\section{The prognostic value of LAG-3 by Cox proportional hazards survival analysis}

Next, we examined OS and DFS using Cox proportional hazards model to determine whether LAG-3 expression could serve as an independent biomarker. A series of factors, including age, gender, T, N, M, clinical stages, CD4 TIL/CD8 TIL, and LAG3 expression, were entered into the univariate Cox regression analysis in Table 3 to assess their impact on the OS and PFS of ESCC patients. Based on this analysis, $\mathrm{T}$ status, $\mathrm{N}$ status, clinical stages, CD4 TIL/CD8 TIL, and LAG-3 expression were found to impact OS and PFS.

The variables demonstrated to be significant in the univariate analysis were further analyzed by multivariate Cox regression analysis. The multivariate analysis demonstrated independent predictors of OS and PFS were LAG-3 expression (OS: HR, 0.724; 95\% CI $0.526-0.995 ; p=0.047$; PFS: HR, $0.714 ; 95 \%$ CI $0.521-0.978 ; p=0.036$ ), T status (OS: HR, $1.625 ; 95 \% \mathrm{CI}$ $1.052-2.510 ; p=0.029 ;$ PFS: HR, $1.749 ; 95 \%$ CI 1.133-2.698; $p=0.012$ ), and $\mathrm{N}$ status (OS: HR, 2.599; 95\% CI 1.563-4.323; $p=0.000$; PFS: HR, 2.704; 95\% CI $1.624-4.500 ; p=0.000$ )(Table 3 ).

Table 3. Univariate and Multivariate analysis with the COX proportional hazards for predictor of OS, and PFS of ESCC patients

\begin{tabular}{|c|c|c|c|c|c|c|}
\hline \multirow{2}{*}{$\begin{array}{l}\text { Prognostic } \\
\text { factors }\end{array}$} & \multicolumn{3}{|c|}{ Univariate } & \multicolumn{3}{|c|}{ Multivatiate } \\
\hline & HR & CI & $\mathbf{P}$ & HR & CI & $\mathbf{P}$ \\
\hline \multicolumn{7}{|l|}{ OS } \\
\hline Gender & 0.891 & $0.655-1.213$ & 0.465 & $\ldots$ & $\ldots$ & $\ldots$ \\
\hline Age & 0.822 & $0.608-1.109$ & 0.200 & $\ldots$ & $\ldots$ & $\ldots$ \\
\hline $\mathrm{T}$ & 1.720 & $1.203-2.459$ & $0.003^{*}$ & 1.625 & $1.052-2.510$ & $0.029 *$ \\
\hline $\mathrm{N}$ & 2.409 & $1.771-3.278$ & $0.000^{*}$ & 2.599 & $1.563-4.323$ & $0.000^{*}$ \\
\hline M & 0.902 & $0.288-2.826$ & 0.860 & & & \\
\hline Stages & 2.082 & $1.541-2.811$ & $0.000^{*}$ & 0.815 & $0.472-1.408$ & 0.463 \\
\hline $\begin{array}{l}\text { CD4 TIL/CD8 } \\
\text { TIL }\end{array}$ & 0.689 & $0.510-0.932$ & $0.015^{*}$ & 0.771 & $0.570-1.044$ & 0.093 \\
\hline LAG-3 & 0.648 & $0.475-0.886$ & $0.006^{*}$ & 0.724 & 0.526-0.995 & $0.047^{*}$ \\
\hline PFS & & & & $\ldots$ & $\ldots$ & $\ldots$ \\
\hline Gender & 0.926 & $0.688-1.247$ & 0.614 & $\ldots$ & $\ldots$ & $\ldots$ \\
\hline Age & 0.832 & $0.626-1.131$ & 0.252 & $\ldots$ & $\ldots$ & $\ldots$ \\
\hline $\mathrm{T}$ & 1.829 & $1.281-2.611$ & $0.001^{*}$ & 1.749 & $1.133-2.698$ & $0.012^{*}$ \\
\hline $\mathrm{N}$ & 2.430 & $1.793-3.292$ & $0.000^{*}$ & 2.704 & $1.624-4.500$ & $0.000^{*}$ \\
\hline M & 0.854 & $0.273-2.675$ & 0.786 & & & \\
\hline Stages & 2.105 & $1.565-2.831$ & $0.000^{*}$ & 0.790 & $0.458-1.362$ & 0.396 \\
\hline $\begin{array}{l}\text { CD4 TIL/CD8 } \\
\text { TIL }\end{array}$ & 0.674 & $0.501-0.907$ & $0.009^{*}$ & 0.761 & $0.564-1.026$ & 0.073 \\
\hline LAG-3 & 0.648 & $0.475-0.886$ & $0.006^{*}$ & 0.714 & $0.521-0.978$ & $0.036^{*}$ \\
\hline
\end{tabular}

\section{Discussion}

Since cancer immunotherapy was named "breakthrough of the year" by Science in 2013, cancer immunology has presented a totally different way of curing cancer by targeting the host immune system instead of tumor cells [20]. Immune checkpoints are the most important immune proteins in regulating self-tolerance and escape from the immune system. Besides the broadly studies of two Immune checkpoints (cytotoxic T lymphocyte-associated molecule- 4 (CTLA-4) and PD-1)[5-7, 21], more attentions have been gained in co-inhibitory receptors, including TIM3, TIGIT, and LAG-3 et al [22].

Sigurd M. Hald reported that intraepithelialLAG-3 and stromal-LAG-3 were both associated with improved Disease-specific Survival (DSS) and OS in NSCLC [14]. Additionally, LAG-3+ TILs are an independent positive prognostic factor in stage I-IIIB NSCLC [14]. In this ESCC cohort, we demonstrated that decreased LAG-3 expression was significantly associated with advanced clinical stages (Table 2). ESCC patients with higher LAG-3 expression was positively correlated with a better OS and PFS, especially in the patients at T1-2 status, N0 status, and early stages (I-II) (Figure 1 and 2). Cox proportional hazards regression analysis showed that LAG-3 is an independent predictor of survival (Table 3). Taken together, our results suggested that LAG-3 plays an important role in the development of ESCC.

LAG-3 has been shown to be presented on $\mathrm{CD} 4+$, CD8+, and NK cells [9]. LAG-3 expression has been reported to correlate with other immune markers [18]. CD8, PD-1, and LAG-3 have been shown a strong correlation in breast cancer [18]. Both PD-1 and LAG-3 are demonstrated as inhibitory receptors, their expression might suggest insufficient immune response [22]. In addition, LAG-3 was mainly expressed in the $\mathrm{CD} 4^{+} \mathrm{CD} 25^{-} \mathrm{T}$ cells, which showed high expression levels of another two inhibitory immune checkpoint protein, PD-1 and TIM3[19]. LAG-3-expressed $\mathrm{CD}^{+}{ }^{+} \mathrm{CD} 25^{-} \mathrm{T}$ cells were detected more in metastasis than in primary tumors, suggested these $\mathrm{T}$ cells might play important roles in cancer immune escape [19]. Here, we showed that LAG-3 expression was significantly associated with the presence of CD4 TIL and CD8 TIL, as well as the ratio of CD4/CD8 TIL (Table 2). The combination of both high LAG-3 expression and high ratio of CD4/CD8 TIL was also presented a longer OS and PFS than both low of them (Figure 1 and 2). Similarly to other reports, the increased numbers of CD4 TILs and CD8 TILs were correlated to good survival in ESCC and NSCLC et al. [4, 23]. Stromal TILs was identified as independently prognostic factor in Stage III-IVa ESCC [24]. Exhausted CD8 TILs contributes to cancer 
progress [25]. Tumor-infiltrating interleukin (IL)-17Aproducing cells (Th17 cells) are mainly CD4 positive and FoxP3 negative [26] in ESCC. Th17 cells were correlated with CD8 and CD1a dendritic cells (DCs) $[26,27]$, enhanced the cytotoxic activities of NK cells [26]. Moreover, the high level of Th17 cells was associated with good prognosis in ESCC patients [27], suggesting that Th17 cells play an important role in immune regulation during ESCC development. In our study, the higher ratio of CD4 TIL/CD8 TIL was associated with a longer survival in our cohort, indicating the dysregulation of CD4 TIL and CD8 TIL in advanced stages of ESCC. Further investigation is needed to demonstrate the percentage of LAG-3 expression on different subtypes of TILs and the clinical effects of blocking LAG-3 in ESCC.

\section{Conclusion}

In summary, we found decreased LAG-3 expression was significantly associated with advanced clinical stages. Furthermore, higher LAG-3 expression was positively correlated with a better survival and LAG-3 is an independent predictor of survival, suggesting that LAG-3 may serve as a useful immune marker for the prognosis of ESCC.

\section{Supplementary Material}

Supplementary figures and tables. http://www.jcancer.org/v09p4287s1.pdf

\section{Acknowledgements}

The authenticity of this article has been validated by uploading the key raw data onto the Research Data Deposit public platform (www.researchdata.org.cn), with the approval RDD number as RDDB2018000373.

\section{Funding}

This work was supported by the Funding Program of Key Project of Science and Technology Program of Guangzhou (201607020038) and National Natural Science Foundation (81572600).The authors disclose no potential conflicts of interest.

\section{Competing Interests}

The authors have declared that no competing interest exists.

\section{References}

1. Smyth EC, Lagergren J, Fitzgerald RC, Lordick F, Shah MA, Lagergren P, et al. Oesophageal cancer. Nature reviews Disease primers. 2017; 3: 17048.

2. Chen W, Zheng R, Baade PD, Zhang S, Zeng H, Bray F, et al. Cancer statistics in China, 2015. CA: a cancer journal for clinicians. 2016; 66: 115-32.

3. Schumacher K, Haensch W, Roefzaad C, Schlag PM. Prognostic significance of activated CD8(+) T cell infiltrations within esophageal carcinomas. Cancer research. 2001; 61: 3932-6.

4. Cho Y, Miyamoto M, Kato K, Fukunaga A, Shichinohe T, Kawarada Y, et al. CD4+ and CD8+ $\mathrm{T}$ cells cooperate to improve prognosis of patients with esophageal squamous cell carcinoma. Cancer research. 2003; 63: 1555-9.
5. Borghaei H, Paz-Ares L, Horn L, Spigel DR, Steins M, Ready NE, et al. Nivolumab versus Docetaxel in Advanced Nonsquamous Non-Small-Cell Lung Cancer. The New England journal of medicine. 2015; 373: 1627-39.

6. Brahmer I, Reckamp KL, Baas P, Crino L, Eberhardt WE, Poddubskaya E, et al. Nivolumab versus Docetaxel in Advanced Squamous-Cell Non-Small-Cell Lung Cancer. The New England journal of medicine. 2015; 373: 123-35.

7. Hamid O, Robert C, Daud A, Hodi FS, Hwu WJ, Kefford R, et al. Safety and tumor responses with lambrolizumab (anti-PD-1) in melanoma. The New England journal of medicine. 2013; 369: 134-44.

8. Zhu X, Lang J. Programmed death-1 pathway blockade produces a synergistic antitumor effect: combined application in ovarian cancer. Journal of gynecologic oncology. 2017; 28: e64.

9. Triebel F, Jitsukawa S, Baixeras E, Roman-Roman S, Genevee C, Viegas-Pequignot E, et al. LAG-3, a novel lymphocyte activation gene closely related to CD4. The Journal of experimental medicine. 1990; 171: 1393-405.

10. Kisielow M, Kisielow J, Capoferri-Sollami G, Karjalainen K. Expression of lymphocyte activation gene 3 (LAG-3) on B cells is induced by $\mathrm{T}$ cells. European journal of immunology. 2005; 35: 2081-8.

11. Huard B, Gaulard P, Faure F, Hercend T, Triebel F. Cellular expression and tissue distribution of the human LAG-3-encoded protein, an MHC class II ligand. Immunogenetics. 1994; 39: 213-7.

12. Xu F, Liu J, Liu D, Liu B, Wang M, Hu Z, et al. LSECtin expressed on melanoma cells promotes tumor progression by inhibiting antitumor T-cell responses. Cancer research. 2014; 74: 3418-28.

13. Kouo T, Huang L, Pucsek AB, Cao M, Solt S, Armstrong T, et al. Galectin-3 Shapes Antitumor Immune Responses by Suppressing CD8+ T Cells via LAG-3 and Inhibiting Expansion of Plasmacytoid Dendritic Cells. Cancer immunology research. 2015; 3: 412-23.

14. Hald SM, Rakaee M, Martinez I, Richardsen E, Al-Saad S, Paulsen EE, et al. LAG-3 in Non-Small-cell Lung Cancer: Expression in Primary Tumors and Metastatic Lymph Nodes Is Associated With Improved Survival. Clinical lung cancer. 2018 May;19(3):249-259.

15. Lee SJ, Jun SY, Lee IH, Kang BW, Park SY, Kim HJ, et al. CD274, LAG3, and IDO1 expressions in tumor-infiltrating immune cells as prognostic biomarker for patients with MSI-high colon cancer. Journal of cancer research and clinical oncology. 2018;144(6):1005-1014.

16. Woo SR, Turnis ME, Goldberg MV, Bankoti J, Selby M, Nirschl CJ, et al. Immune inhibitory molecules LAG-3 and PD-1 synergistically regulate T-cell function to promote tumoral immune escape. Cancer research. 2012; 72: 917-27.

17. Xie J, Wang J, Cheng S, Zheng L, Ji F, Yang L, et al. Expression of immune checkpoints in $\mathrm{T}$ cells of esophageal cancer patients. Oncotarget. 2016; 7: 63669-78.

18. Burugu S, Gao D, Leung S, Chia SK, Nielsen TO. LAG-3+ tumor infiltrating lymphocytes in breast cancer: clinical correlates and association with PD-1/PD-L1+ tumors. Annals of oncology : official journal of the European Society for Medical Oncology. 2017; 28: 2977-84.

19. Ma QY, Huang DY, Zhang HJ, Wang S, Chen XF. Function and regulation of LAG3 on CD4(+)CD25(-) T cells in non-small cell lung cancer. Experimental cell research. 2017; 360: 358-64.

20. Couzin-Frankel J. Breakthrough of the year 2013. Cancer immunotherapy. Science. 2013; 342: 1432-3.

21. Ribas A. Anti-CTLA4 Antibody Clinical Trials in Melanoma. Update on cancer therapeutics. 2007; 2: 133-9.

22. Anderson AC, Joller N, Kuchroo VK. Lag-3, Tim-3, and TIGIT: Co-inhibitory Receptors with Specialized Functions in Immune Regulation. Immunity. 2016; 44: 989-1004.

23. Kinoshita T, Muramatsu R, Fujita T, Nagumo H, Sakurai T, Noji S, et al. Prognostic value of tumor-infiltrating lymphocytes differs depending on histological type and smoking habit in completely resected non-small-cell lung cancer. Annals of oncology : official journal of the European Society for Medical Oncology. 2016; 27: 2117-23.

24. Jiang D, Liu $\mathrm{Y}$, Wang H, Wang H, Song Q, Sujie A, et al. Tumour infiltrating lymphocytes correlate with improved survival in patients with esophageal squamous cell carcinoma. Scientific reports. 2017; 7: 44823.

25. McKinney EF, Smith KG. T cell exhaustion and immune-mediated disease-the potential for therapeutic exhaustion. Current opinion in immunology. 2016; 43: 74-80.

26. Lu L, Pan K, Zheng HX, Li JJ, Qiu HJ, Zhao JJ, et al. IL-17A promotes immune cell recruitment in human esophageal cancers and the infiltrating dendritic cells represent a positive prognostic marker for patient survival. Journal of immunotherapy. 2013; 36: 451-8.

27. Lv L, Pan K, Li XD, She KL, Zhao JJ, Wang W, et al. The accumulation and prognosis value of tumor infiltrating IL-17 producing cells in esophageal squamous cell carcinoma. PloS one. 2011; 6: e18219. 